\title{
Medical Image of the Week: Saddle Pulmonary Embolism
}

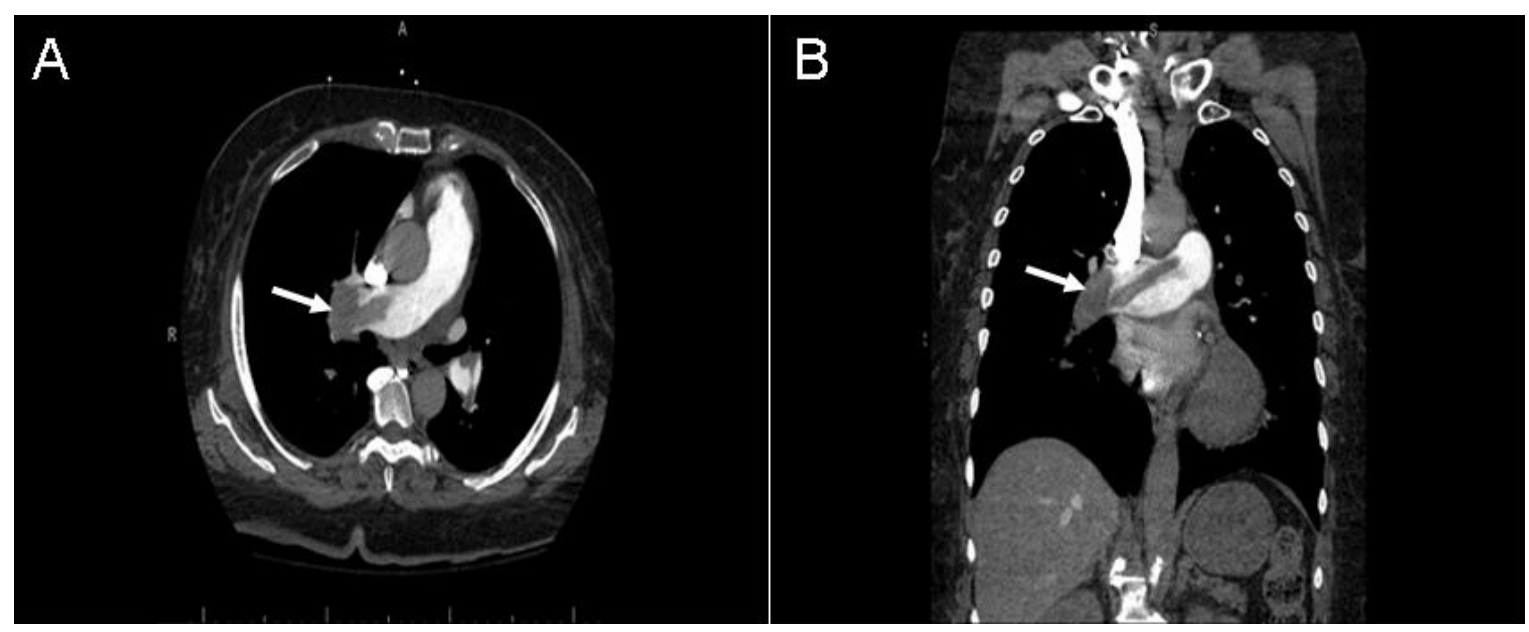

Figure 1. Representative static images from the thoracic computed tomography angiogram demonstrating saddle embolism within bilateral pulmonary arteries with extension into sub-segmental branches (arrows). Panel A: axial CT. Panel

B: coronal CT.

A 66-year-old woman with recent history of left knee surgery and L2-L5 spinal fusion within the past month presented to the Emergency Department (ED) with pleuritic chest pain and shortness of breath for three days. On admission, reported crushing diffuse substernal chest pain worsened to $10 / 10$ on the pain scale on the day of presentation. In the ED, physical examination was remarkable for tachycardia, tachypnea, diaphoresis and hypotension. Initial electrocardiogram was significant for sinus tachycardia with S1Q3T3 pattern. thoracic computed tomography angiogram (CTA) showed saddle pulmonary embolus (PE) with extension into segmental vasculature, right greater than left (Figure 1). A bedside echocardiogram demonstrated diastolic and systolic bowing of the intraventricular septum into the left ventricle. An emergent transthoracic echocardiogram confirmed flattened septum consistent with right ventricle pressure overload with right ventricular systolic pressure of $55 \mathrm{mmHg}+$ central venous pressure (CVP) and reduced left ventricular ejection fraction of $38 \%$. Her hemodynamic condition was stabilized by administering intravenous norepinephrine infusion. Interventional radiology was contacted for an emergent catheter directed thrombolysis. Ten milligrams of tissue plasminogen activator (tPA) was injected into the pulmonary artery with subsequent removable inferior vena cava filter placement. Vasoactive medications were titrated down and stopped four hours following tPA administration and anticoagulation with intravenous heparin was commenced. The patient was discharged from the hospital after initiation of rivaroxaban. 
Saddle pulmonary embolism (SPE) is a form of large pulmonary thromboembolism that straddles the main pulmonary arterial branch at the bifurcation. The incidence of SPE is found in about $2.6 \%$ of the PE cases (1). The choice of diagnostic testing for PE is CTA based on multiple studies demonstrating CTA was both sensitive and specific for PE especially in moderate to high clinical probability PE $(2,3)$. Suspicion for PE in our patient included sedentary status following knee replacement surgery, tachycardia, S1Q3T3 pattern on EKG, evidence for right ventricular strain on echocardiography, and no other diagnosis more likely thus lead to high clinical suspicion of pulmonary embolism with a Well's score for PE of 6 .

Faraz Jaffer, $\mathrm{MD}^{1}$ See Wei Low, $\mathrm{MD}^{1}$ and Sairam Parthasarathy, $\mathrm{MD}^{2}$ ${ }^{1}$ Department of Internal Medicine, Banner - University Medical Center ${ }^{2}$ Department of Allergy, Pulmonary, Critical Care and Sleep Medicine Banner-University Medical Center

Tucson, AZ USA

\section{References}

1. Ryu JH, Pellikka PA, Froehling DA, Peters SG, Aughenbaugh GL. Saddle pulmonary embolism diagnosed by CT angiography: frequency, clinical features and outcome. Respir Med. 2007 Jul;101(7):1537-42. [CrossRef] [PubMed]

2. Van Belle A, Büller HR, Huisman MV, et al. Effectiveness of managing suspected pulmonary embolism using an algorithm combining clinical probability, D-dimer testing, and computed tomography. JAMA. 2006 Jan 11;295(2):172-9. [CrossRef] [PubMed]

3. Stein PD, Fowler SE, Goodman LR, et al. Multidetector computed tomography for acute pulmonary embolism. N Engl J Med. 2006 Jun 1;354(22):2317-27. [CrossRef] [PubMed] 